\title{
An Empiricist View of Managing Globally Distributed Software Development
}

\author{
Narayan Ramasubbu \\ Singapore Management University, Singapore \\ nramasub@smu.edu.sg
}

\begin{abstract}
Software Engineering research is still catching up with the explosive growth in the adoption and proliferation of distributed software development in its many forms. In this talk, I will present the research roadmap I, as an empirical researcher, had taken to investigate distributed software development, and will highlight the key findings and inferences from my exploration. Drawing evidence from more than two hundred large scale distributed software development projects that I had observed in the past five years, I will discuss the challenges faced by distributed software teams along with the way these teams (and their organizations) have responded to the challenges. I will also share my views on the existing gaps, both theoretical and empirical, in software engineering economics literature that need to be bridged to further our understanding of distributed software development. These gaps specifically relate to how software engineering researchers and practitioners accommodate distributedness in project planning, execution, control and reflection activities. Overall, my discussions will call for a new set of governance schemes specifically suited for distributed software development projects, and will lay out a roadmap for empirical software engineers to build one.
\end{abstract}

\section{Short Biography}

Narayan Ramasubbu is an assistant professor at the School of Information Systems at the Singapore Management University. He has a Ph.D. in Business Administration from the University of Michigan, Ann Arbor, and an Electronics and Telecommunications Engineering degree from Bharathiyar University, India. Prior to pursuing the Ph.D., he was a senior developer and product management specialist, first at CGI Inc. and then at SAP AG. His research focuses on software engineering economics, distributed software product development, and software product standardization and customization. His research statement and projects can be viewed at http://www.sis.smu.edu.sg/faculty/infosys/nramasub.asp. 